\title{
Intelligent Driving System for Safer Automobiles
}

\author{
Hideo Inoue ${ }^{1, a)}$ Pongsathorn Raksincharoensak ${ }^{2}$ Shintaro Inoue ${ }^{3}$
}

Received: September 6, 2016, Accepted: November 9, 2016

\begin{abstract}
As an innovation of driver assistance technology, this research aims to develop an "Autonomous Intelligent Driving System" to prevent risk of accidents and enhance driving safety for elderly drivers in order to vitalize current aged society. The proposed system focuses on two key technologies: Risk-predictive driving intelligence model and Shared control between the driver and the assistance system. The first key technology is to embed an experienced driver model for recovering degraded performances of recognition, decision-making and operation of drivers. In the driver assistance system design, the experienced driver model contains knowledge-based "risk-prediction mechanism" to avoid accidents in risky driving situations. For instance, when passing unsignalized intersections with poor visibility, it is known that experienced drivers predict the appearance of sudden-crossing pedestrians or bicycles and then slow down the vehicle when approaching such poor visibility area and also prepare to brake in order to avoid potential collisions that might occur. The second key point is "Shared control." This research does not aim to develop a fully-autonomous driving vehicle for them, but aims to develop an advanced driver assistance system for preventing accidents in the case that the intervention by braking or steering is needed, as well as reducing driving workload. Therefore, to realize good cooperative characteristics between the driver and the system, the shared control concept is applied to optimize the assistance level for braking and steering maneuver, minimizing the interference human driver driving maneuver. The Driving Simulator and the test vehicle are used to verify the effectiveness of the proposed intelligent driving system.
\end{abstract}

Keywords: intelligent driving system, risk prediction, shared control, path planning, elderly driver, automated driving

\section{Introduction}

Recent advances in passive safety and active safety technologies have greatly contributed to a remarkable decrease in traffic fatalities, less than 4,500 fatalities in 2015 , however, the number of road accidents remains high according to national statistics data [1]. By 2030, it is estimated that the ratio of the people older than 65 years will reach $1 / 3$ and the ratio of the latterstage elderly people older than 75 years will reach $1 / 5$ among the populations of Japan [2]. Focusing on the elderly drivers, it is also estimated that half of car drivers older than 60 years will be holding their driving licenses. Based on these facts, accidents involving elderly drivers have been increasing every year owing to their declined physical ability in terms of recognition, decision-making and operation. For instance, the effective visibility range for elderly drivers is relatively narrower than that for young drivers. Based on a driving test of a forward collision warning system using a large driving simulator, it was found that there are many elderly drivers who cannot recognize the collision warning sound produced by the driver assistance system [3]. These facts indicate that in critical situations, an autonomous driving system is one of the promising solutions to prevent collisions involving elderly drivers. Moreover, degraded driving performance reduces the self-confidence of elderly drivers. Most of elderly drivers still need automobiles as a means of mobility

\footnotetext{
Kanagawa Institute of Technology, Atsugi, Kanagawa 243-0292, Japan Tokyo University of Agriculture and Technology, Koganei, Tokyo 1848588, Japan

3 Toyota Motor Corporation, Susono, Shizuoka 410-1193, Japan

a) inoue@eng.kanagawa-it.ac.jp
}

for active social participation as well as to improve their quality of life [4]. To recover their degraded driving performance, this research project aims at considerably improving road safety by allowing autonomous driving control intervention in the last few seconds before an accident involving elderly drivers.

Drive recorders have been used to record real world nearmiss incident data from more than 200 taxi vehicles in Tokyo, Shizuoka, Kyushu, Hokkaido region since 2005. Currently, 110,000 event data are registered in the database system of Tokyo University of Agriculture and Technology [5]. The data has been used for human error analysis and cause and effect chain study of accidents. Based on past studies, there are many cases that current autonomous emergency braking functions cannot avoid crashes such as pedestrians or bicycles dart out from blind spot or occlusion. It is important to predict the hidden risk in driving situation as the experienced driver behave and control the vehicle velocity and direction to a certain value to prepare for unexpected upcoming hazardous events. The applicant has used the concept of "Risk Potential" to express the risk predictive driving behavior of the experienced drivers in theoretical way. The risk potential of the observed obstacles and the hidden obstacles in the driving corridor of the vehicle are sum up to get the whole risk potential on the road. The optimal trajectory is determined by selecting the future trajectory which minimizes the risk potential on the road while securing good ride comfort for the driver.

However, cooperative characteristics during driving between the system and the driver is an important issue. Strong level of control intervention results in good accident avoidance performance but the driver acceptance might be low. Here, the concept 
of Haptic Shared control is also applied to realize good cooperative characteristics between the system and the driver by optimally controlling the force or torque on the accelerator pedal and the steering wheel to induce the driver to drive safer and act like the experienced driver.

The objective of the research is to develop an autonomous driving control which is designed based on the key concept of experienced driver behavior modeling with risk potential prediction in order to prepare for unexpected upcoming hazardous events. In the project study, to make the system be friendly to all types of drivers including elderly drivers, high driver acceptance and social acceptance are also ones of system requirements.

\section{Project Outline of Intelligent Driving Sys- tem}

This project was adopted and started in 2011 as one of the technology innovation projects to vitalize Japan's aging society. The project is sponsored by the Japan Science and Technology Agency (JST). This project is being carried out based on a collaborative framework between universities and industry, including Tokyo University of Agriculture and Technology (TUAT), the University of Tokyo, Kanagawa Institute of Technology (KAIT), the Japan Automobile Research Institute (JARI), Toyota Central R\&D Labs., Inc., and Toyota Motor Corporation [6].

A specific image of driving intelligence can be presented as follows. The first central aspect of driving intelligence is improving the capability of the driver to perceive and recognize the external environment using sensors (i.e., good vision). Another aspect is basic driving planning skills, i.e., the generation of target routes from visual information and the ability to avoid stopped vehicles. Then, based on these basic driving planning skills, the final central aspect is the intelligence to read the future and predict risks. Types of risks extend from visible and explicit items such as bicycles and pedestrians to potential risks such as invisible changes in the environment in front of the vehicle at night or pedestrians jumping out unseen from behind a parked car. Technology to predict these risks has two key points. The first is a model that defines the possibility of a collision several seconds ahead and physically expresses the degree of current risk. There are several methods of achieving this including, the potential field method. This is an important model for generating the optimum driving route with few risks and for controlling the speed of the vehicle. The second point is learning based on empirical driving information. Currently, the determination of risk from the visible environment requires an ontological approach based on large amounts of information. Fusing a physical model and information model in this way to form a prediction model is a new challenge for the automotive industry. The near-miss data accumulated by Tokyo University of Agriculture and Technology is an extremely valuable resource for this information. This data includes risk events such as those in which drivers avoided an accident through emergency braking. Risk prediction may be possible by learning from this data, and incorporating this type of intelligent behavior (such as drivers slowing down after predicting a potential risk) into a machine is a critical part of the technical challenge to enhance safety and to enable automated driving.

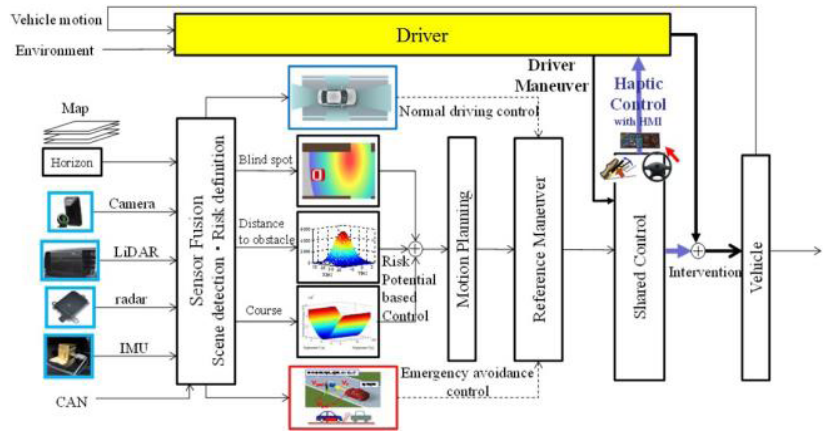

Fig. 1 Conceptual diagram of intelligent driving system.

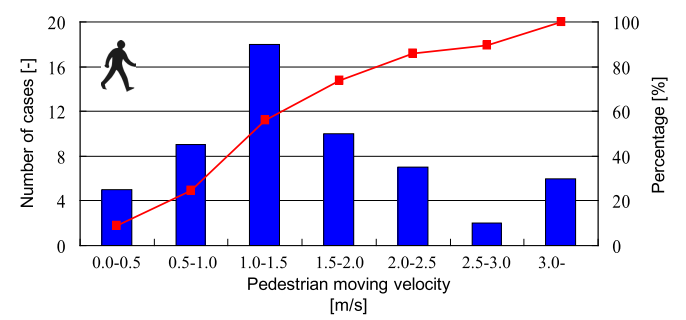

Fig. 2 Pedestrian moving velocity from blind spot from near-miss incident DB analysis.
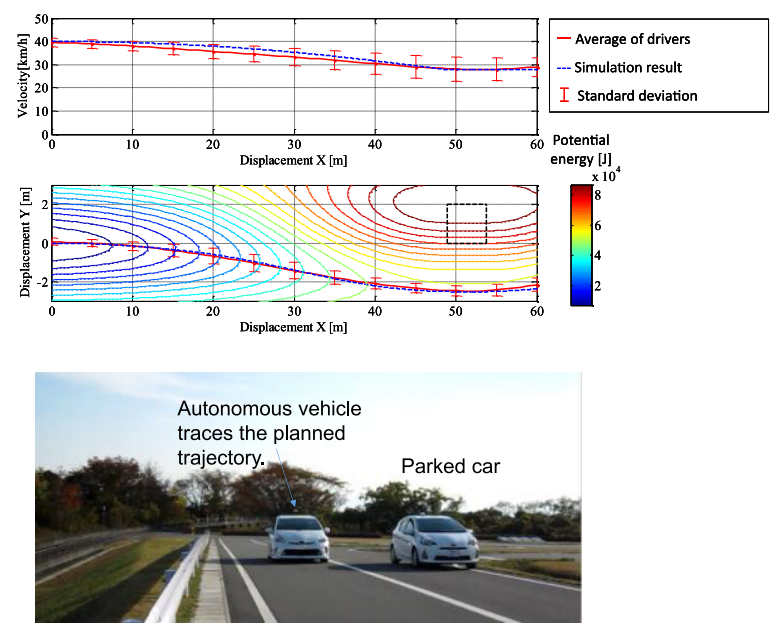

Fig. 3 Motion planning result using risk potential optimization framework (simulation/experiment).

In the conceptual diagram shown in Fig. 1, the normal driving control performs continuously to track a desired path and keep the vehicle at safe speed. By predicting the risk potential based on priori-knowledge as well as experience, the risk-potential based control level is added in order to modify the safe path and safe speed in the time horizon of about 4-5 seconds before the accident risk becomes imminent. The autonomous driving control is designed based on the key concept of experienced driver behavior modeling by assuming the artificial risk potential fields on the driving corridor. The principle of the autonomous vehicle control here is to plan a trajectory within the defined risk potential field, describing the hazardous level of each obstacle or object on the roadway based on knowledge-based risk potential of experienced drivers. In the case that unexpected hazardous event occurs, the emergency avoidance control performs at the Time-to-Collision at about 1 second before the crash occurs as the conventional Autonomous Emergency Braking (AEB) systems work. 


\section{Risk Predictive Driving Technology}

\subsection{Motion Planning and Control System}

Conventional motion planning algorithms in static environment using potential field theory commonly calculate the desired longitudinal and lateral motions, i.e. the vehicle speed and the yaw rate, within the same potential field functions [7]. In the scenario focused in this paper, the static obstacle avoidance maneuver and the vehicle speed control maneuver are calculated separately. The steering maneuver is conducted to minimize the hazard potential by changing the vehicle trajectory not to collide with the static obstacle. The braking maneuver is conducted to slow down the vehicle in order to reach the safe speed not to collide with pedestrians who might appear from behind the obstacle. Based on this design concept, Fig. 4 shows the block diagram of the autonomous driving intelligence system for obstacle avoidance. The lateral motion and the longitudinal motion control problems are formulated independently.

In the lateral motion control, the hazard potential functions with respect to the road boundaries and the obstacle are defined at first. The candidates of the desired yaw rates are defined and consequently the vehicle predicted position with respect to each yaw rate candidate value is calculated by assuming that the vehicle moves at a constant yaw rate within a finite time horizon. In addition, the performance index based on the hazard potentials of road environment is calculated with respect to a number of candidate yaw rates. The desired yaw rate is sequentially selected from the candidate yaw rate values which minimizes the performance index.

In longitudinal control, the risk potential function with respect to the occluded pedestrian is defined using a spring model. The predicted vehicle position within a finite time horizon is calculated in accordance with the candidate longitudinal deceleration values and the performance index considering the risk potential of the occluded pedestrian is calculated with respect to a number of candidate deceleration values. The desired deceleration is sequentially selected from the candidate values which minimizes the performance index.

\subsection{Hazard-Potential Based Lateral Control}

This subsection describes the lateral motion control of the proposed system. The hazard potential used in the lateral motion control system is described by an exponential function. Under the condition that a static obstacle exists in front of the ego vehicle on a straight road, the vehicle path is determined by two factors, i.e. road boundaries and a static obstacle. Actual drivers determined the optimal path reducing the risk of collision with the static obstacle and lane departure. Therefore, artificial potential fields are formulated with respect to the two factors.

The repulsive potential field of road boundaries is defined as follows:

$$
U_{r}(X, Y)=w_{r}\left[1-\exp \left\{-\frac{\left(Y-Y_{r c}\right)^{2}}{2 \sigma_{r}^{2}}\right\}\right]
$$

where, $w_{r}, \sigma_{r}$ are the weight and the variance of the repulsive potential field of road boundaries respectively, and $Y_{r c}$ is the

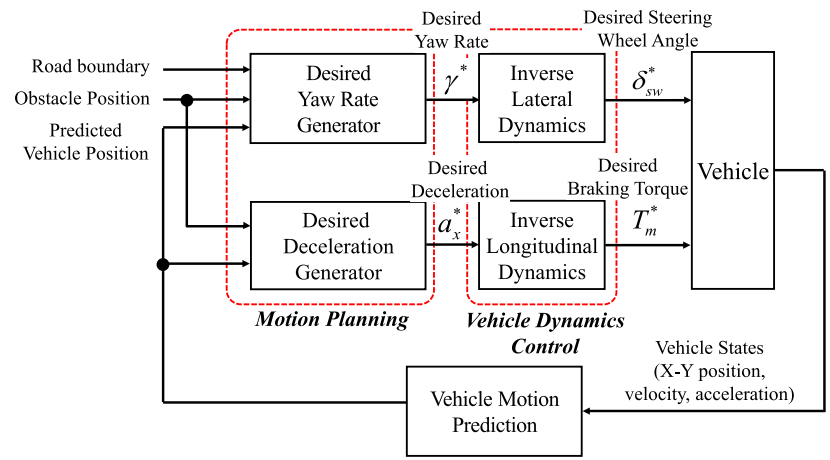

Fig. 4 Block diagram of motion planner and controller of the autonomous driving intelligence system.

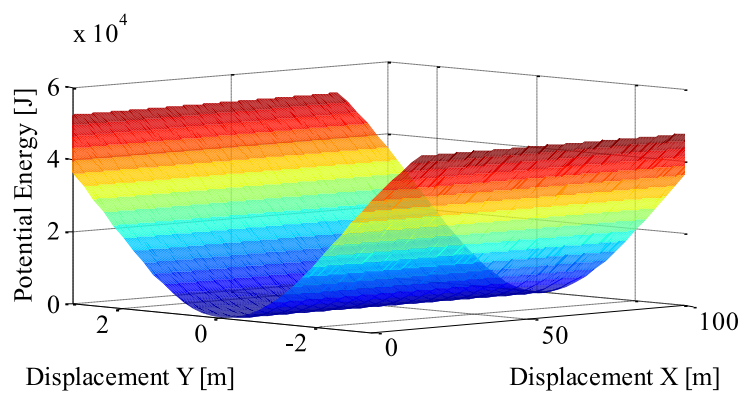

Fig. 5 Repulsive potential field of the road boundaries.

$Y$-coordinate of the road center.

Figure 5 shows the shape of the repulsive potential field of the road boundaries. This potential field has the maximum value at the position of the road boundaries to express risk of lane departure.

Next, the repulsive potential field of the obstacle is defined with respect to the vehicle position as follows:

$$
U_{o}(X, Y)=\left\{\begin{array}{lr}
w_{o} \exp \left\{-\frac{\left(X-X_{o r}\right)^{2}}{\sigma_{o X}^{2}}-\frac{\left(Y-Y_{o}\right)^{2}}{\sigma_{o Y}^{2}}\right\} & \left(X \leq X_{o r}\right) \\
w_{o} \exp \left\{-\frac{\left(Y-Y_{o}\right)^{2}}{\sigma_{o Y}^{2}}\right\} & \left(X_{o r}<X<X_{o f}\right) \\
w_{o} \exp \left\{-\frac{\left(X-X_{o f}\right)^{2}}{\sigma_{o X}^{2}}-\frac{\left(Y-Y_{o}\right)^{2}}{\sigma_{o Y}^{2}}\right\} & \left(X \geq X_{o f}\right)
\end{array}\right.
$$

where, $w_{o}$ indicates the weight of the repulsive potential field of the obstacle, $\sigma_{o X}, \sigma_{o Y}$ are the variances of $X$ direction and $Y$ direction respectively, $X_{o f}, X_{o r}$ indicates the $X$-coordinate at the front end and the rear end of the obstacle respectively. As can be noticed from Eq. (2), the repulsive potential field of the obstacle is defined as an exponential function of $X$ and $Y$.

Figure 6 shows the shape of the repulsive potential field of the static obstacle. This potential field has the maximum value at the position of the obstacle to express risk of collision with the obstacle.

Next, the overall risk potential field is defined as follows:

$$
U_{r i s k}(X, Y)=U_{r}(X, Y)+U_{o}(X, Y)
$$

As can be noticed from Eq. (3), the overall risk potential field is the summation of the repulsive potential field of the road boundaries $U_{r}$ and the repulsive potential field of the obstacle $U_{o}$. $w_{r}, w_{o}, \sigma_{r}, \sigma_{o X}$ and $\sigma_{o Y}$ included in Eqs. (1) and (2) are the 


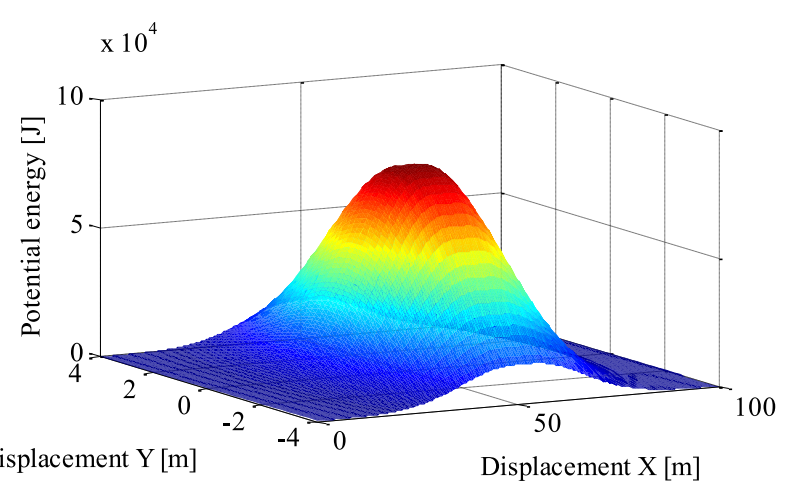

Fig. 6 Repulsive potential field of the static obstacle.

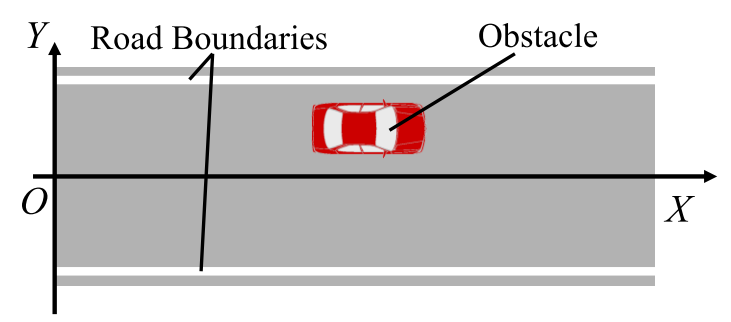

(a) Scenario for risk potential computation

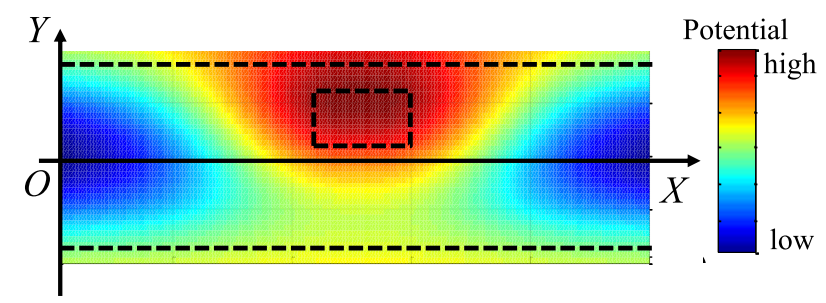

(b) Combined potential field hazard map contour

Fig. 7 Driving scene and map contour of hazard potential.

parameters to vary the shape and height of the risk potential field. These parameters are determined based on the driving data of actual experienced drivers.

In the driving situation shown in Fig. 7 (a), the combined potential hazard contour map is expressed as Fig. 7 (b).

The parameters of the risk potential functions such as the weighting coefficients and the variances of the exponential functions $w_{r}, w_{o}, \sigma_{r}, \sigma_{o X}, \sigma_{o Y}$ are determined from the collected driving data by experienced drivers. By assuming that the experienced drivers select the trajectory which passes through minimum collision risk points of the hazard potential map, the summation of gradient values along the trajectory must be minimum. By applying this idea, the parameter optimization problem can be formulated by using non-linear least-square method. LevenbergMarquardt algorithm is used in this paper. First, the repulsive potential function parameters $w_{r}, \sigma_{r}$ are given as initial values and then the repulsive potential parameters with respect to the parked vehicle $w_{o}, \sigma_{o X}, \sigma_{o Y}$ are optimized by fitting the actual driving data. The following expression indicated in Eq. (4) is used to solve these parameters,

$$
\left[\begin{array}{l}
w_{o} \\
\sigma_{o X} \\
\sigma_{o Y}
\end{array}\right]_{o p t}=\underset{w_{o}, \sigma_{o X}, \sigma_{o Y}}{\arg \min } \sum_{i=1}^{N_{\text {data }}}\left(\frac{\partial}{\partial Y} U_{\text {risk }}\left(X_{e}(i), Y_{e}(i)\right)\right)^{2}
$$

subject to

$$
\left[\begin{array}{c}
w_{r} \\
\sigma_{r}
\end{array}\right]=\text { constant }
$$

where, $X_{e}, Y_{e}$ indicate the vehicle trajectory by the experienced drivers and Ndata indicates the number of trajectories used for parameter identification process.

Next, the repulsive potential function parameters related to the road boundary are determined. By using the parameters $w_{o}, \sigma_{o X}$, $\sigma_{o Y}$ determined from Eq. (4) above, the parameters $w_{r}, \sigma_{r}$ can be calculated by using the following expression.

$$
\left[\begin{array}{c}
w_{r} \\
\sigma_{r}
\end{array}\right]_{o p t}=\underset{w_{r}, \sigma_{r}}{\arg \min } \sum_{i=1}^{N_{\text {data }}}\left(\frac{\partial}{\partial Y} U_{\text {risk }}\left(X_{e}(i), Y_{e}(i)\right)\right)^{2}
$$

subject to

$$
\left[\begin{array}{l}
w_{o} \\
\sigma_{o X} \\
\sigma_{o Y}
\end{array}\right]=\left[\begin{array}{l}
w_{o} \\
\sigma_{o X} \\
\sigma_{o Y}
\end{array}\right]_{o p t}
$$

Next, the calculation of the desired yaw rate is determined by using the above-mentioned potential field functions. Conventional path planning by the potential field method is conducted by calculating the gradient of the potential field at arbitrary vehicle position [7], [8], [9], [10], [11], [12], [13], [14], [15]. However, the problem that the stability of the vehicle motion can be ensured in comparatively higher speed compared to mobile robot speed region and the problem that the vehicle cannot move out from at the local minimum point of the potential field may occur. Trajectory generator can be designed by using model predictive control framework [15], [16], but it includes high complexity in the calculation process and it is difficult to implement for real-time vehicle control. To avoid these problems, the proposed lateral motion control system determines the vehicle yaw rate sequentially by selecting the yaw rate which results in minimum hazard potential with the application of optimal control theory.

As the calculation process, the minimum yaw rate $\gamma_{p \text {,min }}$ and maximum yaw rate $\gamma_{p \text {,max }}$ for the search range of the desired yaw rate and the resolution $\Delta \gamma_{p}$ of the search are given. Next, the predictive yaw rate, which is the candidate of the desired yaw rate $\gamma_{p}(i)$ are defined as follows:

$$
\gamma_{p}\left(i_{y}\right)=\gamma_{p, \min }+\Delta \gamma_{p} \cdot i_{y} \quad\left(i_{y}=0,1,2, \cdots, M_{y}\right)
$$

where, $M_{y}$ indicates the number of yaw rate candidates. When the ego vehicle moves at a constant yaw rate with negligible side slip angle, the predicted vehicle positions $\left(X_{p_{-} y}, Y_{p_{-}}\right)$at a time horizon $t_{p_{-} y}$ with the command yaw rate $\gamma_{p}$ can be calculated as follows:

$$
\left[\begin{array}{l}
X_{p_{-} y}\left(i_{y}, j_{y}\right) \\
Y_{p_{-} y}\left(i_{y}, j_{y}\right)
\end{array}\right]=\left[\begin{array}{l}
X_{e}(t)+\int_{t_{0}}^{t_{0}+t_{p-y}\left(j_{y}\right)} V(t) \cos \left(\psi(t)+\gamma_{p i} t\right) d t \\
Y_{e}(t)+\int_{t_{0}}^{t_{0}+t_{p-y}\left(j_{y}\right)} V(t) \sin \left(\psi(t)+\gamma_{p i} t\right) d t
\end{array}\right]
$$

where, $\psi$ indicates the yaw angle. Next, the performance index for determination of the desired yaw rate is defined as follows: 


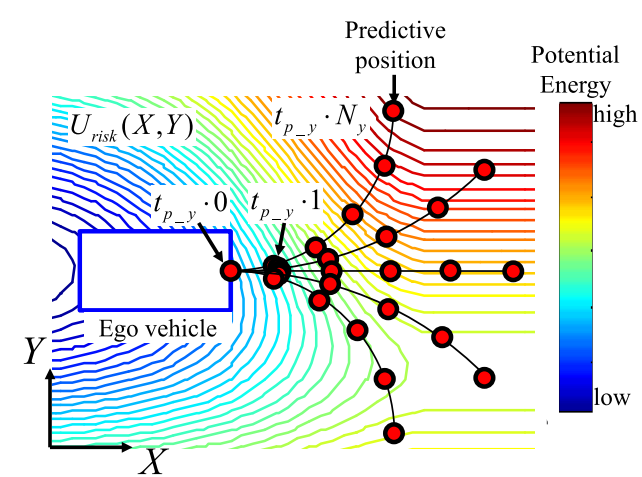

Fig. 8 Schematic diagram of the predictive position and the risk potential.

$$
J_{y}\left(i_{y}\right)=\sum_{j_{y}=1}^{N_{y}}\left[U_{Y}\left(X_{p_{-} y}\left(i_{y}, j_{y}\right), Y_{p_{-} y}\left(i_{y}, j_{y}\right)\right)+q \gamma_{p i}^{2}\right]
$$

where $q$ indicates the weighting coefficient of yaw rate input. Figure 8 shows the schematic diagram of the predictive position and the risk potential. The feature of the performance index $J_{y}$ is that it contains the intermediate variable as the potential field function not vehicle state variables. The performance index consists of the summation of the hazard potential field at the vehicle predicted position and the square of the command yaw rate within a finite time horizon. The performance index $J_{y}$ is calculated according to each command yaw rate candidate and the desired yaw rate $\gamma^{*}$ is determined as the value that results in the minimum value of the performance index $J_{y}$. This calculation process is conducted at each sampling time.

Next, the calculation of the desired steering wheel angle is described. If the side slip angle at the gravity center and the change rate of the vehicle velocity are negligible, the desired steering wheel angle $\delta_{s w}{ }^{*}$ can be expressed using the equivalent linear two-wheel vehicle model as follows:

$$
\delta_{s w}^{*}(t)=n\left(1+K V(t)^{2}\right) \frac{l}{V(t)} \gamma^{*}(t)
$$

where, $K$ indicates the stability factor, $n$ is the steering gear ratio. Since the frequency of the steering maneuver is low in this driving scenario, the dynamic characteristics of the yaw rate with respect to the steering wheel angle input is neglected.

\subsection{Risk-Potential Based Longitudinal Control}

This subsection describes the longitudinal motion control of the proposed system. The risk potential function used in the longitudinal motion control system is described as a spring model. Under the condition that a poor visibility cornor caused by occlusions such as a parking vehicle exists, actual drivers reduce the collision risk with respect to a darting-out pedestrian by early braking based on hazard anticipation knowledge. Although road infrastructure such as V2X communication can be effectively used to know the existence of pedestrians and then prevent collisions, this study aims to apply experienced driver hazard anticipatory characteristics to the design of intelligent driving system without requiring communication systems. To further decrease the number of pedestrian fatalities, the risk assessment algorithm which considers the collision risk including such occluded objects is essential. Based on this fact, an artificial potential field

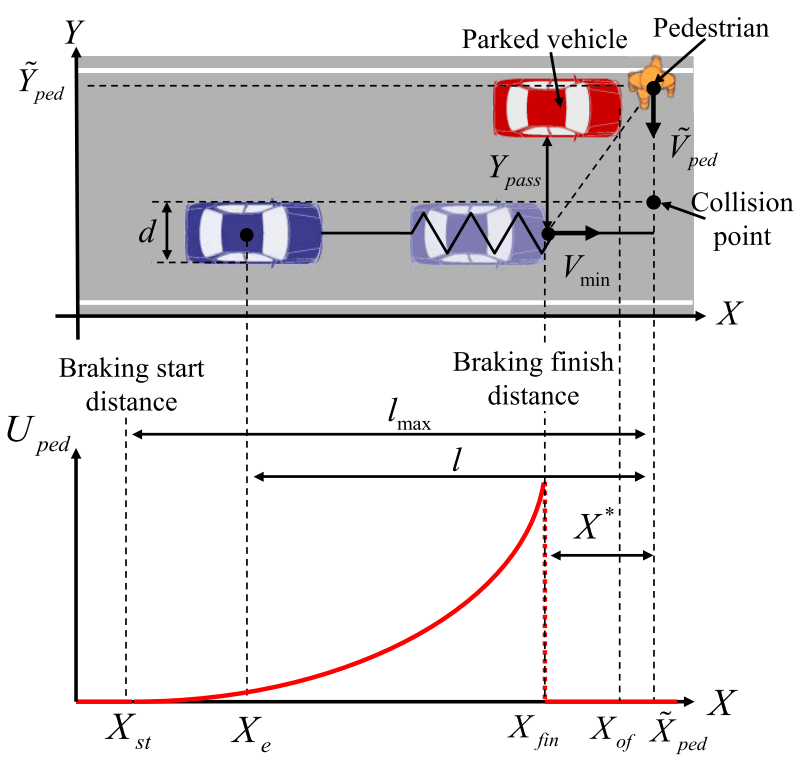

Fig. 9 Repulsive risk potential field of the occluded pedestrian.

regarding the occluded pedestrian is also introduced in the motion planning computation algorithm. The repulsive potential field of the occluded pedestrian $U_{\text {ped }}$ is defined as follows:

$$
U_{p e d}=\frac{1}{2} k_{p e d}\left[l_{\max }-l(t)\right]^{2} \quad \text { if } \quad X^{*}<l(t)<l_{\max }
$$

where, $k_{\text {ped }}$ indicates the spring constant of the repulsive potential of the occluded pedestrian, $l$ indicates the relative distance between the pedestrian moving axis and the ego vehicle. In addition, $l_{\max }$ and $X^{*}$ indicate the maximum and minimum distances with respect to the occluded pedestrian which causes risk potential, respectively. $l_{\max }$ refers to the braking start distance and $X^{*}$ refers to the braking finish distance.

Next, Eq. (11) is obtained based on the law of energy conservation between the potential energy and the kinetic energy of the vehicle. In other words, the kinetic energy must be reduced as the potential energy increases in the artificial riks potential field.

$$
\frac{1}{2} m V(t)^{2}+\frac{1}{2} k_{p e d}\left[l_{\max }-l(t)\right]^{2}=\frac{1}{2} m V_{\min }^{2}+\frac{1}{2} k_{p e d}\left(l_{\max }-X^{*}\right)^{2}
$$

where, $m$ indicates the vehicle mass and $V_{\min }$ indicates the desired minimum velocity which is determined based on the driving data of experienced drivers.

Based on Eq. (11), the spring constant is determined as follows:

$$
k_{p e d}=\frac{m\left(V_{\min }^{2}-V(t)^{2}\right)}{\left[l_{\max }-l(t)\right]^{2}-\left[l_{\max }-X^{*}\right]^{2}}
$$

The spring constant varies sequentially based on the velocity and the relative distance. Figure 9 shows the shape of the repulsive risk potential field of the occluded pedestrian. To calculate the value of the spring constant, the terminal vehicle velocity $V_{\min }$ must be given. The terminal vehicle velocity $V_{\min }$ can be theoretically determined from the stopping distance equation and the geometrical relationship of the vehicle and the pedestrian as follows:

[Stopping distance]

$$
X^{*}=V_{\min } \tau+\frac{V_{\min }^{2}}{2 a_{\max }}
$$




$$
\begin{aligned}
& \frac{1}{2 a_{\max }} V_{\min }^{2}+\tau V_{\min }-X^{*}=0 \\
& V_{\min }=\frac{-\tau+\sqrt{\tau^{2}+\frac{2 X^{*}}{a_{\max }}}}{\frac{1}{a_{\max }}} \\
& =a_{\max }\left(-\tau+\sqrt{\tau^{2}+\frac{2 X^{*}}{a_{\max }}}\right)
\end{aligned}
$$

where, $a_{\max }$ denotes the maximum deceleration applied for stopping, $\tau$ the braking reaction time with respect to the pedestrian recognition and $X^{*}$ the position where the velocity is lowest. The position where the velocity is lowest is dependent on the distance to the parked vehicle $Y_{\text {pass }}$ by solving the following equations.

$$
\begin{aligned}
& \frac{\widetilde{Y}_{p e d}-\frac{d}{2}}{V_{p e d}}=\frac{X^{*}}{V_{\min }} \\
& \widetilde{Y}_{p e d}=\frac{\widetilde{X}_{p e d}-X_{f i n}}{X_{\text {of }}-X_{f i n}} Y_{p a s s}
\end{aligned}
$$

Equation (16) is obtained by using the similarity of triangles in the pictorial diagram of Fig. 7. By substituting Eq. (16) into Eq. (15), the $X^{*}$ can be expressed as a function of the distance to the parked vehicle $Y_{\text {pass }}$. In Eq. (15), $V_{\text {ped }}$ is defined as the average walking speed of pedestrian in crash-relevant events, using the pedestrian motion analysis in the Eq. (12).

Next, the calculation of the desired deceleration is described. The maximum deceleration $a_{x, \max }$ and the resolution $\Delta a_{x}$ for the search of the desired deceleration are given and the a number of decelerations which are the candidates of the desired deceleration $a_{x}\left(i_{x}\right)$ are defined as follows:

$$
a_{x}\left(i_{x}\right)=\Delta a_{x} \cdot i_{x} \quad\left(i_{x}=0,1,2, \cdots, M_{x}\right)
$$

where, $M_{x}$ indicates the number of acceleration candidates. When the ego vehicle moves only the longitudinal direction, the predicted vehicle position $X_{p_{-} x}\left(i_{x}, j_{x}\right)$ at a time horizon $t_{p_{-} x}\left(j_{x}\right)$ with respect to the deceleration $a_{x}\left(i_{x}\right)$ are calculated as follows:

$$
\begin{aligned}
& X_{p_{-} x}\left(i_{x}, j_{x}\right) \\
& =X_{e}(t)+V(t) \cdot t_{p_{-} x}\left(j_{x}\right)+\frac{1}{2} a_{x}\left(i_{x}\right) \cdot t_{p_{-} x}\left(j_{x}\right)^{2}
\end{aligned}
$$

Next, the performance index $J_{x}$ for the determination of the desired deceleration is defined as follows:

$$
J_{x}\left(M_{x}\right)=\sum_{j_{x}=1}^{N_{x}}\left(U_{p e d}\left(X_{p_{-} x}\left(i_{x}, j_{x}\right)\right)+r a_{x}^{2}\right)
$$

where, $r$ indicates the weight of the command longitudinal deceleration input. This performance index $J_{x}$ is expressed as the summation of the risk potential at the predictive position and the square of the predictive deceleration. The performance index $J_{x}$ is calculated at the several predictive decelerations and the desired deceleration $a_{x}{ }^{*}$ is determined as the value which minimizes the performance index $J_{x}$. This calculation process is sequentially conducted at each sampling time as same as the lateral motion control.

The braking torque command of the vehicle $T_{m}{ }^{*}$ in order to

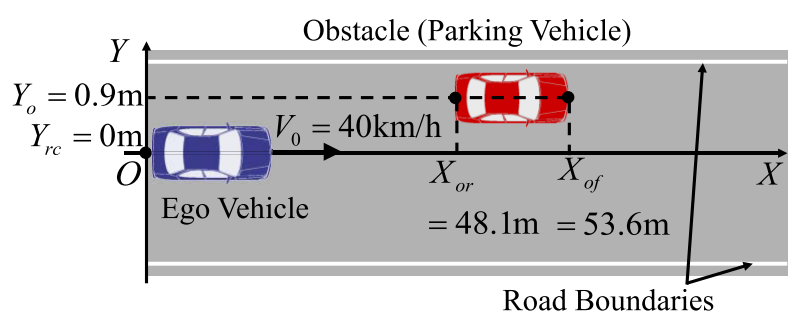

Fig. 10 Driving scenario specifications.

Table 1 Parameters of the lateral vehicle control.

\begin{tabular}{|c|c|c|}
\hline Symbol & Value & Unit \\
\hline$w_{r}$ & $7.41 \times 10^{4}$ & - \\
\hline$\sigma_{r}$ & 2.40 & - \\
\hline$w_{o}$ & $8.99 \times 10^{4}$ & - \\
\hline$\sigma_{o X}$ & 27.8 & - \\
\hline$\sigma_{o Y}$ & 3.05 & - \\
\hline$\gamma_{p \_ \text {min }}$ & -0.50 & $\mathrm{rad} / \mathrm{s}$ \\
\hline$\gamma_{p \_\max }$ & 0.50 & $\mathrm{rad} / \mathrm{s}$ \\
\hline$\Delta \gamma_{p}$ & 0.01 & $\mathrm{rad} / \mathrm{s}$ \\
\hline$q$ & 200 & - \\
\hline
\end{tabular}

Table 2 Parameters of the longitudinal vehicle control.

\begin{tabular}{|c|c|c|}
\hline Symbol & Value & Unit \\
\hline$l_{\max }$ & 50 & $\mathrm{~m}$ \\
\hline$a_{p \_x, \max }$ & -3.0 & $\mathrm{~m} / \mathrm{s}^{2}$ \\
\hline$\Delta a_{p \_x}$ & 0.1 & $\mathrm{~m} / \mathrm{s}^{2}$ \\
\hline$r_{x}$ & 60 & - \\
\hline
\end{tabular}

achieve the desired longitudinal deceleration $a_{x}{ }^{*}$ is determined by using the inverse dynamics of the vehicle longitudinal motion combined with the one-wheel rotational motion model. The longitudinal slip ratio is assumed to be zero.

$$
T_{m}^{*}=\frac{1}{2}\left(\frac{J+m r_{w}^{2}}{r_{w}} a_{x}^{*}+F_{R} r_{w} \cdot \operatorname{sgn}(V)\right)
$$

where, $J$ denotes the moment of inertia of the tire-wheel, $r_{w}$ the effective radius of the tire, and $F_{R}$ the driving resistance.

\subsection{Validation of the Proposed Motion Planning and Con- trol}

\subsubsection{Driving Scenario}

The simulation was conducted to verify the effectiveness of the proposed motion planning and control system. The simulation scenario is shown in Fig. 10. The simulation was conducted on the straight road in which the parking vehicle existed. The front end and the rear end of the parking vehicle were set as far as $53.6 \mathrm{~m}$ and $48.1 \mathrm{~m}$ from the start point respectively. Additionally, the parking vehicle was located at a lateral distance of $0.9 \mathrm{~m}$ from the road center. The ego vehicle was running at a speed of $40 \mathrm{~km} / \mathrm{h}$ at first. As a vehicle model in the simulation, the 4 -wheel vehicle model was used. The simulation result was compared with the driving data of the selected experienced drivers. Additionally, the control parameters of the longitudinal motion and the lateral motion control are shown in Table $\mathbf{1}$ and Table 2. 

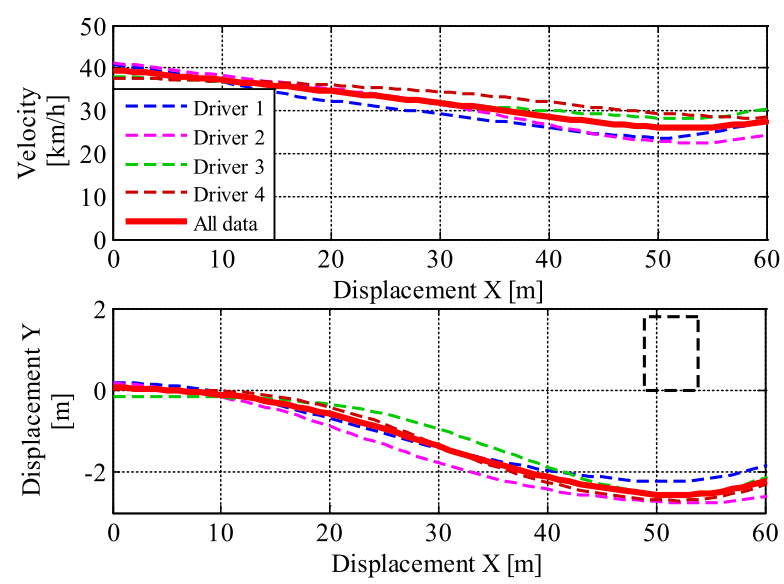

Fig. 11 Driving data of experienced drivers.
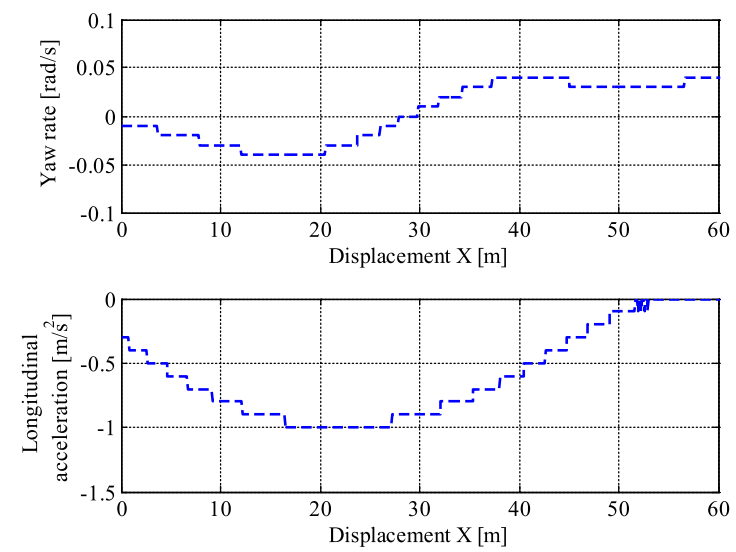

Fig. 12 Motion planning computation results.

\subsubsection{Driving Data of Experienced Drivers}

The driving data of experienced drivers were collected. Four drivers who are test drivers of automotive manufacturers and driving instructors who get well-trained in safety driving were selected as the experienced drivers. They drove under the same condition as the scenario, indicated in Fig. 10, seven times and the data of the vehicle velocity and the trajectory were acquired. The average trajectory and the average speed of each driver as well as the average of all driver data are shown in Fig. 11. In this paper, the effectiveness of the proposed system was verified by comparing the simulation result with the average values of all driver data.

\subsubsection{Simulation Results and Discussions}

The desired vehicle motion calculated by the motion planning algorithm is shown in Fig. 12 and the velocities and the vehicle trajectories of the simulation and experienced drivers are shown in Fig. 13. Figure 14 shows the braking distances with and without the risk prediction. The braking distance in Fig. 14 is calculated assuming that the maximum jerk and the response time are $12 \mathrm{~m} / \mathrm{s}^{3}$ and $0.1 \mathrm{~s}$ considering the recognition time of the pedestrian detection sensor. The drawn lines indicate the required braking distance with respect to the velocity, at the time instant that the pedestrian darts out from the space behind the parked vehicle, in order to avoid collision when the maximum deceleration for stopping is given $(0.2 \mathrm{G}, 0.4 \mathrm{G}, 0.6 \mathrm{G}$ and $0.8 \mathrm{G})$. The distance of the pedestrian appearance is set at $8.0 \mathrm{~m}, 10.0 \mathrm{~m}, 12.0 \mathrm{~m}$ and $14.0 \mathrm{~m}$. The marks plotted in the graph indicate the vehicle

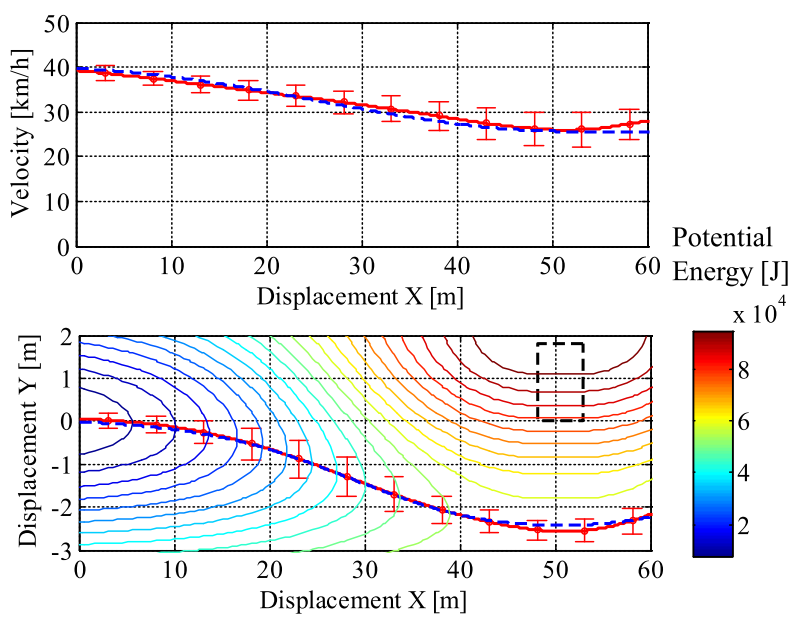

Fig. 13 Comparison of actual experienced driver data (red line) and simulation (blue line) with the proposed motion planning and control system (Standard deviation of the experiment data by experienced driver is also shown with red bars).

: With risk potential prediction $\square$ : Without risk potential prediction

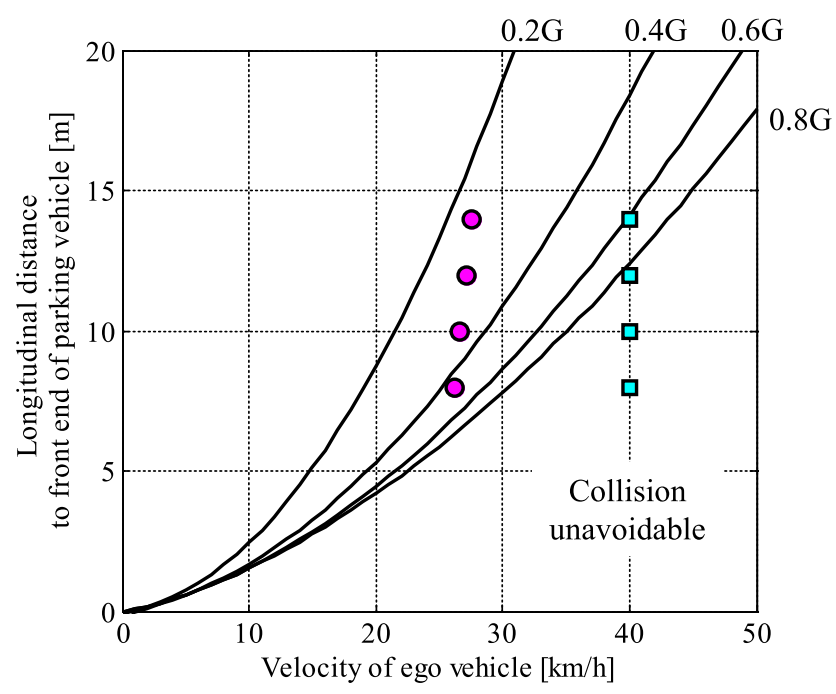

Fig. 14 Required deceleration for pedestrian collision avoidance by braking.

velocity at each condition in the case that the risk prediction is conducted (circles) and not conducted (squares). The vehicle velocity becomes lower when the risk prediction is considered in the vehicle motion planning. The position of each mark also indicates the magnitude of the required maximum deceleration in order to avoid collision. The mark below the line of $0.8 \mathrm{G}$ means that the required deceleration is higher than $0.8 \mathrm{G}$ which exceeds the braking capability of the vehicle on dry road friction condition. In other words, the collision is unavoidable. In this paper, the simulation finish point was defined as $X=60 \mathrm{~m}$ as the proposed system focused on the parking-vehicle overtaking and the collision avoidance with occluded objects.

As can be noticed from Fig. 11 and Fig. 12, the ego vehicle was able to avoid the parking vehicle with the stable behavior. Moreover, the simulation results about both the vehicle velocity and trajectory closely match the driving data of the experienced drivers. Therefore, the proposed autonomous driving system with 
combination of risk potential field and optimal control theories are feasible to express the actual anticipatory driving characteristics of the experienced drivers. In addition, as can be noticed from Fig. 14, the ego vehicle equipped with an autonomous emergency braking (AEB) without pedestrian risk potential prediction cannot avoid the collision even with $0.8 \mathrm{G}$ if a pedestrian dart out at the distance between the ego vehicle and the front end of the obstacle of $12 \mathrm{~m}$. On the other hand, the ego vehicle with the risk-potential based motion planning and control can avoid the collision with $0.4 \mathrm{G}$ in the same situation. Moreover, the ego vehicle with the control can avoid the collision with $0.8 \mathrm{G}$ even if a pedestrian darts out at the distance between the ego vehicle and the front end of the obstacle of $8 \mathrm{~m}$. In this fact, the proposed riskpotential based control system can effectively enhance pedestrian crossing collision avoidance performance.

\section{Shared Control}

People have used machines as soon as the trustworthiness of the machine has been established. At the same time, as mentioned above, people may change from being willing drivers to unwilling drivers due to fatigue or the driving conditions. Elderly people often feel unease about driving due to self-awareness of physical deterioration. One of the potential roles of an intelligent machine may be to reassure and enable the elderly to drive safely. The right to drive cannot be taken away simply on the grounds of age. Freedom of mobility is one of the inherent merits of a car and driving is in itself a miraculous act. One universal aspect of people using machines is the inspiration that this use generates, and the instinct that connects this inspiration to human vitality and motivation. The use of information through smart phones has become widespread in the modern age. If information contains the value of "knowing," then objects must also contain the opposite values of "seeing, touching, and feeling." However, this is not a discussion about which is better. The key point for both information and objects is how to communicate the context considered by a machine to the person using it. Ignoring the issue of the human-machine interface (HMI), it is necessary to identify whether the results meet or exceed the expectations of the person using the machine, since this is the root of trust between the two. A machine must predict the result, communicate the context of this action to the user and ensure understanding. From this standpoint, smart phones are ahead of automated driving technologies. This is still a weak point for vehicle driving. A machine that can communicate and express a route or a situational awareness judged to be the optimal option as rationally as possible through the steering wheel, accelerator pedal, or brakes, is finally approaching the capabilities of a horse. In this case, it is not acceptable to add new interfaces that increase the burden of driving. Can a machine perfectly identify the context of driving and communicate this to the driver? That is what is required of driving intelligence with shared control.

This chapter investigates a new shared control system which combines haptic steering guidance torque and Direct YawMoment Control (DYC). The proposed system can potentially solve the interference problem, as DYC is used to control the lateral dynamics as an independent control input [17], [18], [19],

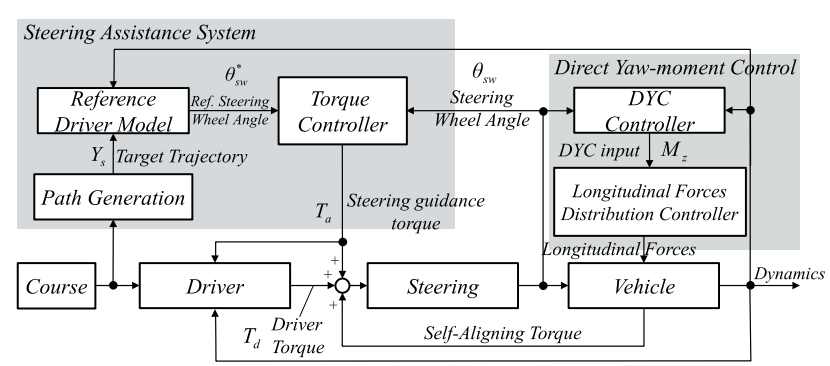

Fig. 15 Human-machine shared control system.

[20], [21], [22], [23], [24], [25], [26], [27], [28], [29].

\subsection{Shared Steering Control System Design}

Figure 15 shows the proposed system. The steering assistance system aims to induce the driving behavior of elderly drivers to be close to that of a reference driver model (Eq. (21)).

$$
\theta_{s w}^{*}=\frac{h_{s}}{1+\tau_{n s} s}\left\{Y_{s}-\left(Y_{c}+T_{p s} V \psi\right)\right\}
$$

where, $\theta_{s w}{ }^{*}$ denotes the reference steering wheel angle, $h_{s}$ denotes the reference driver gain, $\tau_{n s}$ denotes the reference time constant, $Y_{s}$ denotes the reference lateral displacement at the preview point, $Y_{c}$ denotes the lateral displacement of center of gravity, $T_{p s}$ denotes the reference preview time, $V$ denotes the vehicle speed, $\Psi$ denotes the yaw angle. The haptic steering guidance torque control law is expressed as Eq. (22).

$$
T_{a}=K_{a}\left(\theta_{s w}^{*}-\theta_{s w}\right)
$$

where, $T_{a}$ denotes the steering guidance torque, $K_{a}$ denotes the steering guidance gain, $\theta_{s w}$ denotes the steering wheel angle. When steering wheel angle $\theta_{s w}$ does not correspond to the reference steering wheel angle $\theta_{s w}{ }^{*}$, the steering guidance torque $T_{a}$ is provided to drivers as a haptic torque from steering wheel, to indicate the steering direction that he/she should apply to drive along the desired path.

The control law of DYC is expressed as Eq. (23).

$$
M_{Z}=K_{m z} \times \frac{\theta_{s w}}{n}
$$

where, $M_{z}$ denotes the DYC input, $K_{m z}$ denotes the DYC gain, $n$ denotes the gear ratio. There are two aims in the design of DYC. The first effect is to reduce self-aligning torque. When self-aligning torque is decreased, driver torque and steering guidance torque are decreased consequently. The second effect is to improve the vehicle responsiveness with respect to the steering angle. According to these effects, the proposed system can reduce the degree of the interference between the driver torque and the steering guidance torque $T_{a}$.

\subsubsection{Driver Model}

This section describes the driver model defined. Equation (24) expresses the driver model with steering torque input (Yuhara, N. et al. 1997) [30].

$$
T_{d}=\frac{1}{1+\tau_{v} s}\left[H\left\{Y_{d}-\left(Y_{c}+T_{p} V \psi\right)\right\}\right]+\frac{1}{1+\tau_{h} s}\left(K_{h} T_{a}\right)
$$

where, $T_{d}$ denotes the driver torque, $\tau_{v}$ denotes the driver visual time constant, $H$ denotes the driver compensatory gain based on 

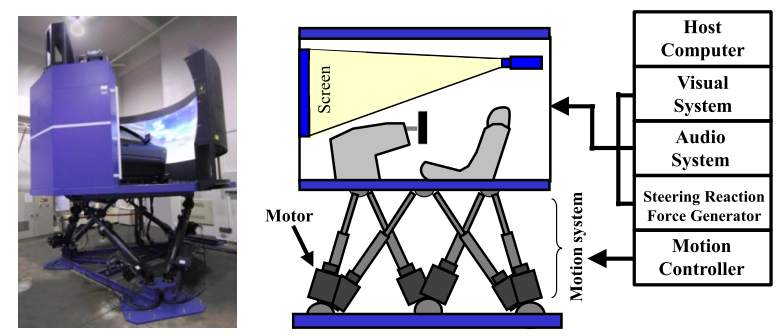

Fig. 16 TUAT driving simulator.

the visual information, $Y_{d}$ denotes the driver target lateral displacement, $T_{p}$ denotes the driver preview time, $\tau_{h}$ denotes the driver haptic time constant, $K_{h}$ denotes the driver compensatory gain based on the haptic information. The first term on the right side in the Eq. (24) indicates the torque with respect to the visual feedback information, whereas the second term indicates the torque with respect to the haptic steering information.

\subsubsection{Steering and Vehicle}

The steering model is used in the control model coupling with the above driver model with steering torque input. The steering equation of motion is expressed as Eq. (25).

$$
\theta_{s w}=\frac{1}{J_{s} s^{2}+C_{s} s}\left(T_{d}+T_{m}-\frac{T_{S A T}}{n}\right)
$$

where, $J_{s}$ denotes the moment of inertia of the steering wheel, $C_{S}$ denotes the viscous damping coefficient of steering wheel side, $T_{m}$ denotes the motor torque of the steering, $T_{S A T}$ denotes the selfaligning torque. The motor torque of the steering $T_{m}$ is expressed as Eq. (26).

$$
T_{m}=K_{p s} \times \frac{T_{S A T}}{n}+T_{a}
$$

where, $K_{p s}$ denotes the coefficient of electric power steering. In this model, the complicated power steering mechanism and the torsional stiffness of the steering model are not considered. Moreover, the self-aligning torque $T_{S A T}$ is transferred from the front tires to the steering shaft directly through the steering gear.

The linear equivalent two-wheel vehicle model is used. The parameters of the vehicle model are defined assuming a mid-size vehicle.

\subsection{Driving Simulator Experiment for Shared Control Sys- tem Evaluation}

\subsubsection{TUAT Driving Simulator}

Figure 16 shows TUAT driving simulator used in this experiment. TUAT driving simulator consists of a host computer, a visual system, an audio system, a steering system and a hexapod motion cueing controller. The driving simulator is equipped with the same driver operational interfaces as a real vehicle. The host computer calculates the vehicle behavior base on the input of driver operational interfaces and consequently generates the driving screen image. The hexapod motion cueing system is operated based on the calculated vehicle dynamics states.

\subsubsection{Experimental Condition}

The experiment was conducted by using TUAT driving simulator to evaluate the proposed shared control system (The ethical approval were obtained). Eight elderly drivers whose average

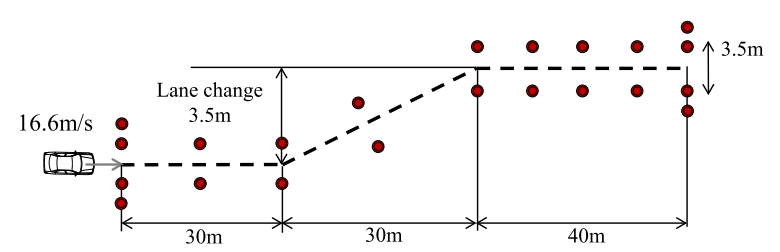

Fig. 17 Driving course of single lane change.

Table 3 Simulation conditions.

\begin{tabular}{|c|c|c|}
\hline Type & $\boldsymbol{K}_{\boldsymbol{a}}[\mathbf{N m}]$ & $\boldsymbol{K}_{\boldsymbol{m} \boldsymbol{z}}[\mathbf{N m}]$ \\
\hline Without control & 0 & 0 \\
\hline Steering control & 4 & 0 \\
\hline Steering+DYC control & 4 & $4 \times 10^{4}$ \\
\hline
\end{tabular}
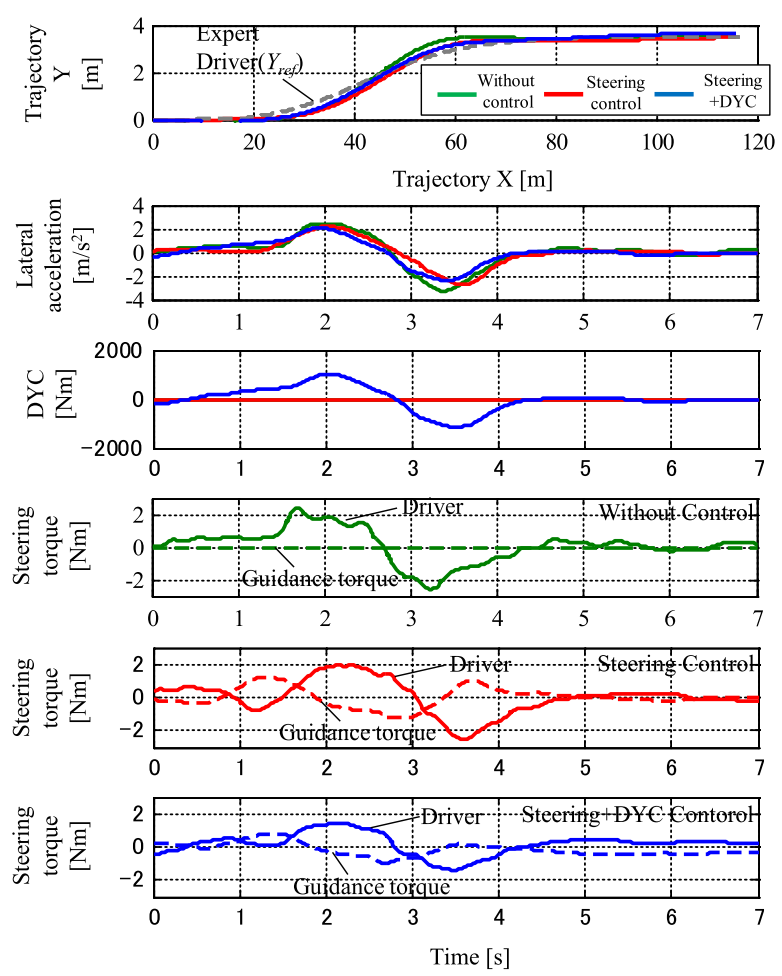

Fig. 18 Experimental results of single lane change (Driver G).

age was 68 years old were employed to drive the simulator ( $4 \mathrm{fe}$ males, 4 male. They usually drive their individual cars with daily scene.) The driving course which is a single lane change is shown in Fig. 17. A vehicle is running at a constant speed of $16.6 \mathrm{~m} / \mathrm{s}$. The control conditions are shown in Table 3. There are "Without control", "Steering control", and "Steering+DYC control." The subjects were instructed to change a lane by using the steering guidance torque $T_{a}$. Meanwhile, since only steering guidance torque $T_{a}$ cannot accomplish a single lane change, the subjects were instructed to conduct proactive maneuver of steering wheel. During the experiments, the subjects were not informed about the control conditions.

\subsubsection{Experimental Results}

The effectiveness of DYC is confirmed in six drivers (A, C, E, F, G, and H) among eight drivers. Figure 18 shows the experimental results by Driver $\mathrm{G}$.

In the graph, $0 \mathrm{~m}$ of trajectory $\mathrm{X}$ refers to $0 \mathrm{~s}$ of time in the horizontal axis. When "Steering control" is compared with the case "Without control", the trajectory Y (the first row) was improved 


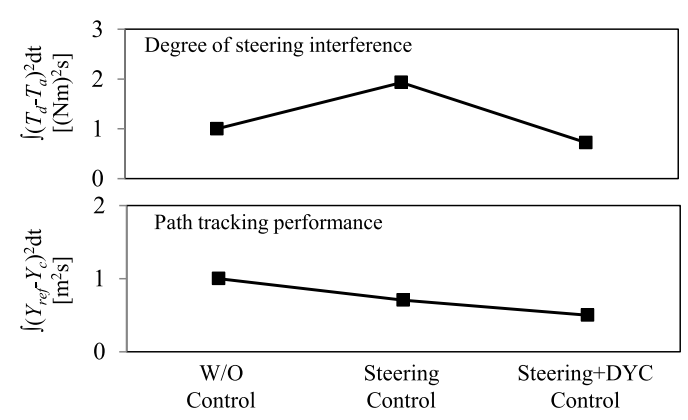

Fig. 19 Square integral values of steering torque difference and deviation from desired path (Driver G, Normalized W/O control).

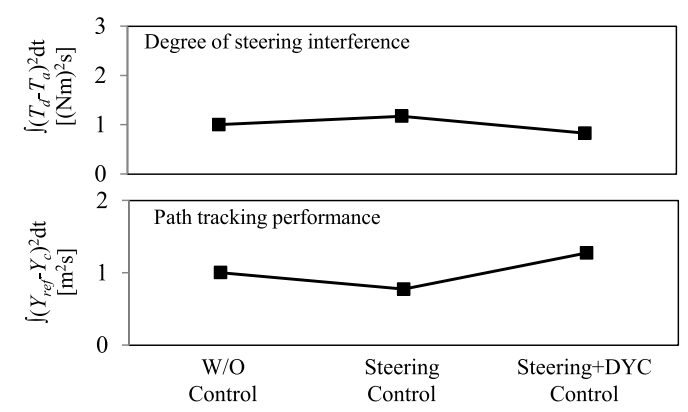

Fig. 20 Square integral values of steering torque difference and deviation from desired path (Driver D, Normalized W/O control).

as the overshoot is reduced in the region from $50 \mathrm{~m}$ to $70 \mathrm{~m}$. Focusing on the steering torque (the fourth and fifth row), it is confirmed that the driver torque $T_{d}$ was obviously improved to be smoother. This result indicates that the steering guidance torque $T_{a}$ is effective to driver $\mathrm{G}$.

The effectiveness of DYC is verified by experiments. As "Steering+DYC control" reduces the amount of driver torque $T_{d}$ and steering guidance torque $T_{a}$, the reduction of the interference between the human and steering assist can be achieved (the sixth row). Figure 19 shows the experimental results by Driver $G$ in which the upper figure indicates the square integral values of the difference between driver torque $T_{d}$ and steering guidance torque $T_{a}$, the lower figure indicates the square integral values of the trajectory of the vehicle $Y_{c}$ deviation with respect to the target trajectory of the expert driver $Y_{\text {ref }}$. Where, the integral time is from $0 \mathrm{~s}$ to $7 \mathrm{~s}$. "Steering+DYC control" performs better path tracking performance with lower steering guidance level. Since similar control effectiveness of DYC is confirmed in the above six drivers, it is verified that the proposed haptic shared steering control system combined with DYC has positive shared control characteristics among the elderly drivers.

Figure 20 shows the square integral values by driver D. Comparing "Steering+DYC control" with "Steering control," the torque interference is slightly reduced showing the same tendency in the case of driver G. On the other hand, the path tracking performance becomes worse compared with the steering control. Since the subjects were instructed to change a lane by using the steering guidance torque $T_{a}$ before starting the experiments, it is considered that driver $\mathrm{D}$ also tried to change his maneuver by using the steering guidance torque $T_{a}$. From this viewpoint, driver D may not understand the assistance system intervention and then apply the steering guidance torque $T_{a}$ inadequately. For these drivers, displaying the target path or improving the DYC

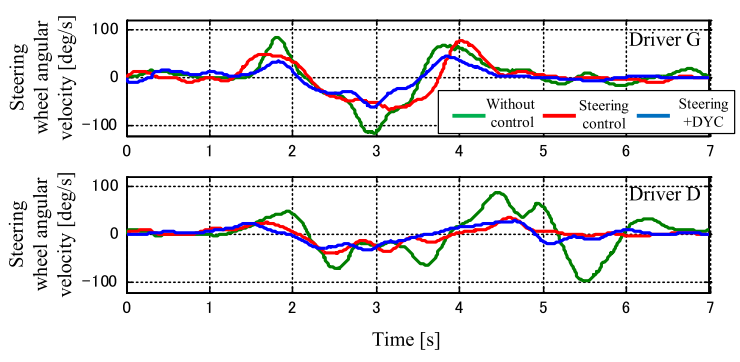

Fig. 21 Time series data of steering wheel angular velocity.

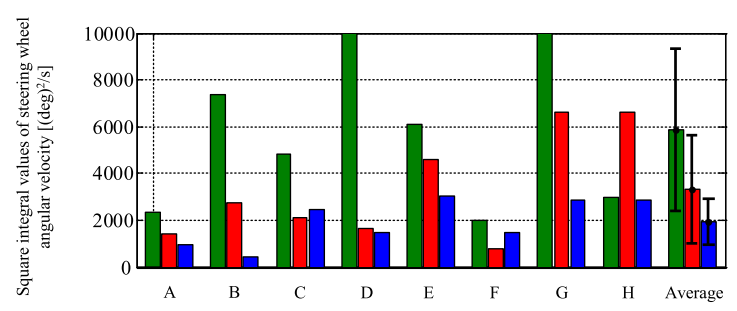

Fig. 22 Square integral values of steering wheel angular velocity.

law may be more effective. We will conduct more detailed study in the future works.

\subsection{Discussion}

\subsubsection{Steering Wheel Angular Velocity Evaluation}

To evaluate the handling quality, steering wheel angular velocity is discussed. Figure $\mathbf{2 1}$ shows the time series data of steering wheel angular velocity by driver $\mathrm{G}$ and driver $\mathrm{D}$. When "Steering control" is applied, the steering angular velocity of each driver was reduced compared to "Without control." Since this result indicates that the maneuver of a driver is improved to be smoother, the effectiveness of steering guidance torque $T_{a}$ is confirmed. This tendency is enhanced when "Steering+DYC control" is applied. This tendency comes from the effects that DYC improves the vehicle responsiveness with respect to the steering angle, and a driver can conduct path tracking in more relaxed manner. Figure 22 shows the square integral values of steering wheel angular velocity among all drivers. Where, the integral time is from $0 \mathrm{~s}$ to $7 \mathrm{~s}$. It is confirmed that except driver $\mathrm{H}$, the square integral values of all drivers was reduced when steering guidance torque $T_{a}$ was applied. It is also confirmed that the standard deviation was smaller when DYC was applied. These results indicate that drivers can conduct a single lane change with the proposed system in smooth manner.

\subsubsection{Driver Model Identification}

To analysis the change of driving behavior, the parameters of driver model are identified using the experimental results obtained in the previous section. The identified lateral driver model is Eq. (24). The identification chooses the parameters to minimize the error $E$ which is defined with Eq. (27) by using Eq. (24) and the experimental results.

$$
E=\int_{t_{1}}^{t_{2}}\left(\hat{T}_{d}-T_{d}\right)^{2} d t
$$

where, $E$ denotes the error values regarding with square integral values of the driver torque, $\hat{T}_{d}$ denotes the estimated driver torque, $t_{1}$ denotes the start time of the integral region, $t_{2}$ denotes the end time of the integral region. In this study, $t_{1}=0 \mathrm{~s}$ and $t_{2}=7 \mathrm{~s}$ 


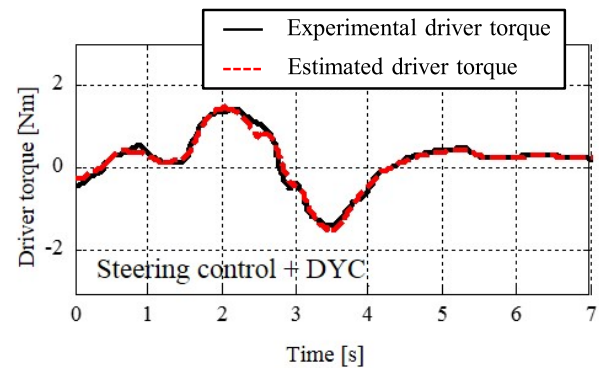

Fig. 23 Relationship between estimated driver torque and that of experiment (Driver G).

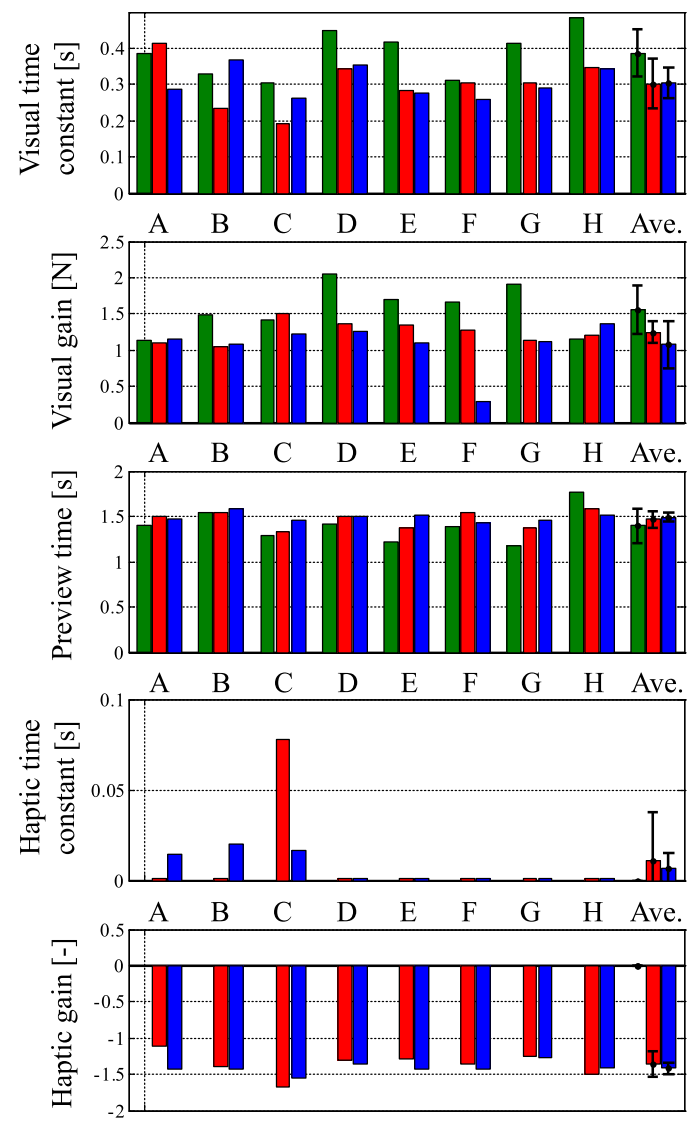

Fig. 24 Identified parameters of all subjects.

is selected. Figure 23 shows the relationship between the estimated driver torque $\hat{T}_{d}$ and the experimental driver torque $T_{d}$ by driver G in the case of "Steering+DYC control". The estimated driver torque $\hat{T}_{d}$ is calculated by using the simulation model in the previous section with the identified parameters of the driver model. Since the estimated driver torque $\hat{T}_{d}$ fits with the measurement value, the estimated parameters are valid for discussions.

Figure $\mathbf{2 4}$ shows the identified parameters among all drivers. It is particularly notable that the haptic time constant $\tau_{h}$ is very small, on the other hand, the visual time constant $\tau_{v}$ is large. Furthermore, it is found that steering guidance torque $T_{a}$ can reduce the visual time delay compared to "without control." These results indicate that the steering guidance torque $T_{a}$ can compensate the time delay in visual feedback. This characteristic is potential to be effective to elderly drivers in reducing the time delay in steering operation during path tracking.

The effectiveness of DYC is confirmed in the visual gain $H$ and the haptic gain $K_{h}$. Comparing "Steering + DYC control" with "Steering control," drivers tend to reduce the visual gain $H$ and emphasize the haptic gain $K_{h}$. This tendency implies that the drivers rely on the steering guidance torque $T_{a}$ more strongly in the case of "Steering+DYC." Further analysis will be conducted in future works to evaluate the relationship between DYC and the change of the driver-vehicle closed loop control characteristics.

\section{Conclusions}

This paper has described two key technologies for an intelligent driving system for safer automobiles. The first is Riskpredictive driving intelligence model and the second key point is 'Shared control.'

An intelligent driving system by embedding the risk potential prediction knowledge of experienced drivers can enhance the driving safety like the obstacle avoidance performance. A typical dangerous scenario in urban environment that a pedestrian suddenly darts out from a blind corner, i.e., behind a parked car, with short time margin to collision is focused. First, the potential field functions of the static road environment and the virtual pedestrian are defined for the lateral and the longitudinal motion control respectively. The hazard potential field for the lateral motion control is determined considering the risk of collision with an obstacle and the risk of lane departure. The risk potential for the longitudinal motion control is determined to express the collision risk with an occluded object such as a pedestrian. Next, the optimal control problem is formulated by taking the potential field functions into the account. The command values for the lateral and the longitudinal control are sequentially determined by solving the formulated optimization problems. Finally, the validity of the proposed control system is verified by comparing the computer simulation results with the actual driving data by experienced drivers. As the result, it has been shown that the proposed autonomous driving system with the combination of potential field theory and the optimal control theory are feasible to express the actual driving of the experienced drivers. From the theoretical collision avoidance analysis, the proposed motion planning and control with risk potential prediction shows superior collision avoidance performance to the conventional automatic emergency braking (AEB) system.

Next, a new shared control system which combines haptic steering guidance torque together with Direct Yaw-Moment Control (DYC) has been investigated to obtain good path tracking performance and human-machine cooperative characteristics. It is verified that the proposed system performs better path tracking performance with lower steering guidance level by the experiment of the driving simulator. Furthermore, the parameters of driver model are identified using the above experimental results, and it is clarified that the steering guidance torque can compensate the time delay in visual feedback of elderly drivers.

As a future plan, the effectiveness of the proposed motion planning and control will be verified by using a prototype experimental vehicle equipped with sensors and the vehicle control system. Driver acceptance issues will be also studied.

Acknowledgments This study has been conducted as a part of the research project "Autonomous Driving Intelligence System to Enhance Safe and Secured Traffic Society for Elderly Drivers" 
grated by Japan Science and Technology Agency (JST). The authors would like to thank the agency for providing financial support to conduct this research.

\section{References}

[1] Traffic Bureau, National Police Agency: Traffic Accidents Statistics in Japan in 2013, National Police Agency, available from 〈http://www.npa.go.jp/toukei/index.htm\#koutsuu〉 (accessed 2014-0526).

[2] Cabinet Office, Government of Japan, White Paper of the Aged Society in 2013 (2013).

[3] Satomi, Y., Murano, T., Aga, M. and Yonekawa, T.: A characteristic analysis of driving behavior to rear-end collision warning using a driving simulator, Proc. 18th JSME Conference on Transportation and Logistics (TRANSLOG), pp.283-286 (2009).

[4] Nagai, M. and Inoue, H.: Research into ADAS with Autonomous Driving Intelligence for Future Innovation, Proc. Chassis Tech Plus 2014, Munich, Germany (2014).

[5] Raksincharoensak, P.: Drive Recorder Database for Accident/Incident Study and Its Potential for Active Safety Development, FOT-NET Workshop, Tokyo, Japan (2013).

[6] Inoue, H., Nagai, M., et al.: Driving Intelligence System to Enhance Safe and Secured Traffic Society for Elderly Drivers, 2014 JSAE Annual Congress (Spring), Yokohama, Japan (2014).

[7] Khatib, O.: Real-Time Obstacle Avoidance for Manipulators and Mobile Robots, International Journal of Robotics Research, Vol.5, No.1, pp.90-98 (1986).

[8] Kaneko, T., Kuriyagawa, Y. and Kageyama, I.: Calculation Algorithm of Control Target for Autonomous Heavy Vehicle under Mixed Traffic Development of Decision-making Algorithm at Merging Sections, Proc. 2013 JSAE Annual Congress (Autumn), No.144-13, pp.1-6 (2013).

[9] Gerdes, J.C., Rossetter, E.J. and Saur, U.: Combining Lanekeeping and Vehicle Following with Hazard Maps, Vehicle System Dynamics, Vol.36, No.4-5, pp.391-411 (2001).

[10] Reichardt, D. and Schick, J.: Collision Avoidance in Dynamic Environments Applied to Autonomous Vehicle Guidance on the Motorway, Proc. IEEE Intelligent Vehicles Symposium, Paris, France, pp.1-6 (1994).

[11] Wolf, M.T. and Burdick, J.W.: Artificial Potential Functions for Highway Driving with Collision Avoidance, Proc. 2008 IEEE International Conference on Robotics and Automation, Pasadena, California, USA, pp.3731-3736 (2008).

[12] Noto, N., Okuda, H., Tazaki, Y., Inagaki, S. and Suzuki, T.: Obstacle Avoidance Assisting System Based on Personalized Potential Field, Proc. SICE Annual Conference, Tokyo, Japan, pp.476-481 (2011).

[13] Kim, K., Kim, B. and Yi, K.: Integrated Vehicle Motion Control of Automated Vehicles for Integrated Risk Management in Dynamic Driving Environment, Proc. 12th International Symposium on Advanced Vehicle Control (AVEC), pp.706-711 (2014).

[14] Bauer, E. et al.: PRORETA3: An Integrated Approach to Collision Avoidance and Vehicle Automation, Automatisierungstechnik, Vol.60, No.12, pp.755-765 (2012).

[15] Kim, W., Kim, D., Yi, K. and Kim, H.J.: Development of a PathTracking Control System Based on Model Predictive Control Using Infrastructure Sensors, Vehicle System Dynamics, Vol.50, No.6, pp.100-1023 (2012).

[16] Wongwaiwit, P., Raksincharoensak, P. and Michitsuji, Y.: Analysis on Pedestrian and Bicycle Behavior in Unsignalized Intersection Based on Near-Miss Incident Database, Proc. 20th JSME Transportation and Logistics Conference, pp.19-22 (2011).

[17] Forsyth, B.A.C. and Maclean, K.E.: Predictive haptic guidance: Intelligent user assistance for the control of dynamic tasks, IEEE Trans. Visualization and Computer Graphics, Vol.12, No.1, pp.103-113 (2005).

[18] Flemisch, F., Heesen, M., Hesse, T., Kelsch, J., Schieben, A. and Beller, J.: Towards a dynamic balance between humans and automation, Cognition, Technology and Work, Special Issue on Humanautomation Coagency, Vol.14, No.1, pp.3-18 (2012).

[19] Abbink, D.A., Mulder, M. and Boer, E.: Haptic Shared Control: Smoothly shifting control authority, Cognitive Technology Work, Vol.14, pp.19-28 (2012).

[20] Katzourakis, D., Olsson, C., Lazic, N. and Lidberg, M.: Driver Steering Override Strategies for Steering Based Active Safety Systems, Proc. 2nd International Symposium on Future Active Safety Technology Towards Zero-Traffic Accidents (FAST-zero '13), Paper no. 20134591, pp.1-7 (2013).

[21] Saleh, L., Chevrel, P., Claveau, F., Lafay, J.-F. and Mars, F.: Shared Steering Control Between a Driver and an Automation: Stability in the Presence of Driver Behavior Uncertainty, IEEE Trans. Intelligent Transportation Systems, Vol.14, No.2, pp.974-981 (2013).

[22] Hirokawa, M., Uesugi, N., Furugori, S., Kitagawa, T. and Suzuki, K.: Effect of haptic assistance on learning vehicle reverse parking skills, IEEE Trans. Haptics, Vol.7, No.3, pp.334-44 (2014).

[23] Nishimura, R., Wada, T. and Sugiyama, S.: Haptic Shared Control in Steering Operation Based on Cooperative Status Between a Driver and a Driver Assistance System, Journal of Human-Robot Interaction, Vol.4, No.3, pp.19-37 (2015).

[24] Balachandran, A., Brown, M., Elien, S.M. and Gerdes, J.C.: Predictive Haptic Feedback for Obstacle Avoidance Based on Model Predictive Control, IEEE Trans. Automation Science and Engineering, Vol.13, No.1 (2016).

[25] Iwano, K., Raksincharoensak, P. and Nagai, M.: Study on Shared Driving Characteristics between Driver and Collision Avoidance Steering Control Using Driving Simulator, 12th International Symposium on Advanced Vehicle Control (2014).

[26] Seo, J., Choi, J. and Yi, K.: Torque Vectoring for Collision Avoidance of Automated Driving Vehicle using Robust Model Predictive Control, Proc. AVEC '14, No.20149208, pp.35-40 (2014).

[27] Yamakado, M., Nagatsuka, K. and Takahashi, J.: A yaw-moment control method based on a vehicle's lateral jerk information, Vehicle System Dynamics, Vol.52, No.10, pp.1233-1253 (2014).

[28] Abe, M.: Vehicle Handling Dynamics, Theory and Application Butterworth-Heinemann, pp.218-229 (2015).

[29] Raksincharoensak, P., Shino, M. and Nagai, M.: Motion Control of Micro-Scale Electric Vehicle by DYC Considering Lane Marker Information, 8th IEEE International Workshop on Advanced Motion Control, pp.47-52 (2004).

[30] Yuhara, N. et al.: An Advanced Steering System with Active Kinesthetic Feedback for Handling Qualities Improvement, Vehicle System Dynamics, Vol.27, No.5-6, pp.327-355 (1997).

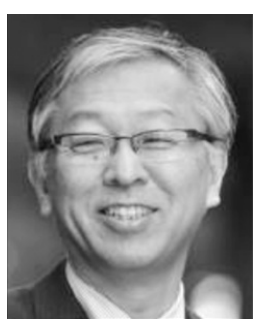

Hideo Inoue was graduated from Waseda University in 1978 and engaged in Toyota Motor Corporation as General Manager of Integrated System Engineering, et al. He is now engaged in Kanagawa Institute of Technology as Professor from this April.

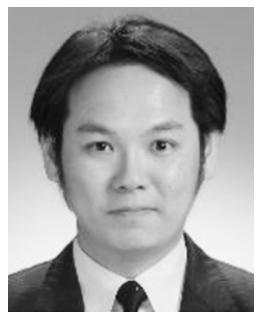

Pongsathorn Raksincharoensak received his Doctor degree in Engineering from Tokyo University of Agriculture and Technology (TUAT) in 2005. He is currently an associate professor at department of mechanical systems engineering at TUAT. His research interests include advanced driver assistance systems and vehicle dynamics control systems.

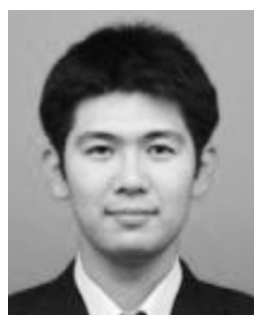

Shintaro Inoue was graduated from Tokyo Institute of Technology and entered Toyota Motor Corporation in 2006. He now researches and develops the advanced driver assistance system based on the steering haptic shared control system. 